\title{
Calculation of critical flow depth using method of algebraic inequality
}

\author{
Tiejie Cheng ${ }^{1}$, Jun Wang ${ }^{1}$, Jueyi Sui ${ }^{2 *}$ \\ ${ }^{1}$ School of Civil Engineering, Hefei University of Technology, 193 Tunxi Road, Hefei, Anhui, China. Email: tj-cheng@qq.com \\ 2 Environmental Engineering, University of Northern British Columbia, 3333 University Way, Prince George, BC, Canada. \\ * Corresponding author. E-mail: Jueyi.sui@unbc.ca
}

\begin{abstract}
To calculate the critical depth and the least specific energy of steady non-uniform flows in open channels, one has to solve the polynomial equations. Sometimes, the polynomial equations are too difficult to get them solved. In this study, the theory of algebraic inequality has been used to derive formulas for determining the critical depth and the least specific energy of a steady non-uniform flow in open channel. The proposed method has been assessed using examples. Results using this new method have been compared to those using other conventional methods by engineers and scientists. It is found that the proposed method based on algebraic inequality theory not only makes the calculation process to be easy, but also gives the best calculation results of the critical depth and the least specific energy of a steady nonuniform flow.
\end{abstract}

Keywords: Critical depth; Mean value inequality; Steady non-uniform flow; Specific energy; Algebraic inequality.

\section{INTRODUCTION}

In practice, the critical depth of steady non-uniform flow in open channel is an important variable for engineers to determine optimal cross section (Vatankhah, 2013). Some crosssectional shapes are often chosen by river engineers in engineering design, such as trapezoidal shape and U-shape, since flow conditions in channels having such cross sections are relatively less complicated in addition to being easily constructed (Liu and $\mathrm{Xu}, 2016$ ). However, for the steady non-uniform flow in channels having such cross sections, it is not easy to determine the critical depth since either polynomial equations or transcendental equations have to be solved. Normally, either the curve-fitting method or trial-and-error method is used to solve the polynomial equations and transcendental equations. Engineers have to put a lot of efforts to solve polynomial equations or transcendental equations. Also, the calculation processes by means of both curve-fitting method and trial-and-error method are multifarious with relatively low accuracy.

The least specific energy and the critical depth of the steady non-uniform flow in open channel have attracted much attention of researchers. Up to date, some methodologies have been proposed for calculating the least specific energy and the critical depth. By introducing following factors $x=m h_{k} / b$ and $k=4 m b^{-1}\left(\alpha Q^{2} g^{-1} b^{-2}\right)^{1 / 3}$, formula have been developed by means of the identical transformation of the basic formula for determining the critical depth in open channels with a trapezoidal cross section (Swamee, 1993; Swamee and Rathie, 2005; Swamee, et al., 1999; Wang, 1998; Zhao, et al., 2009). Li et al. (2010) converted the U-shape cross section into one rectangular part plus two triangular parts. Based on this approach, Li et al. (2010) developed the formula for calculating the critical depth for flow in a channel having an U-shape cross section. By replacing factors in the formulas usually used for calculating the critical depth, Zhang and Li (2012) derived an iterative formula for calculating the critical depth by means of the algebraic transmutation method. Up to date, researchers have improved the calculation accuracy for determining the critical depth by means of different methodologies. However, dimensionless variables have to be solved prior to determining the critical depth. Afterward, based on the calculated dimensionless variables, the critical depth can be determined. Overall, the existing methods for calculating the critical depth are either cumbersome or inaccurate. In the present study, we introduce a new technique to calculate the critical depth easily and accurately. The theory of algebraic inequality is applied to develop formulas for determining the critical depth for steady non-uniform flow in open channels.

\section{CONVENTIONAL HYDRAULIC CALCULATION METHOD FOR STEADY NON-UNIFORM STEADY FLOW IN OPEN CHANNELS}

Chosen the elevation at the channel bed as the reference datum, the hydraulic calculation for steady non-uniform flow in an open channel can proceed. At any individual cross section, the specific energy (defined as the energy per unit weight of fluid) can be determined using the following formula ( $\mathrm{Wu}$, 2008),

$$
E_{s}=h \cos \theta+\frac{\alpha v^{2}}{2 g}=h \cos \theta+\frac{\alpha Q^{2}}{2 g A^{2}}
$$

where, $E_{S}$ is the specific energy of flow at the designated cross section $(\mathrm{m}) ; h$ is flow depth (m); $\theta$ is the slope angle of the channel bed (inclined to the horizontal plane); $\alpha$ is the kinematic energy coefficient; $v$ is the average flow velocity $(\mathrm{m} / \mathrm{s})$; $Q$ is the flow discharge $\left(\mathrm{m}^{3} / \mathrm{s}\right)$; and $A$ is cross-sectional area of flow $\left(\mathrm{m}^{2}\right)$. If the specific energy of flow at the designated cross section approaches the minimum, the flow depth corresponding to the least specific energy is defined as the critical depth $\left(h_{K}\right)$. Conventionally, the least specific energy and critical depth of flow at the designated cross section are calculated by neglecting the slope of channel bed and the kinematic energy coefficient, namely, $\cos \theta=1$ and $\alpha=1.0$. Then, the first derivative of Eq. (1) with respect to $h$ is as follows,

$$
\frac{d E_{s}}{d h}=\frac{d}{d h}\left(h+\frac{Q^{2}}{2 g A^{2}}\right)=1-\frac{Q^{2}}{g A^{3}} \frac{d A}{d h}
$$

The width of water surface $(B)$ can be expressed as $d A / d h$ $=B$; for the least specific energy of flow, let $d E_{S} / d h=0$, then, 
$\frac{A_{k}^{3}}{B_{k}}=\frac{Q_{k}^{2}}{g}$

Eq. (3) is very important, since it is the basis for many researchers to study the critical depth of a steady non-uniform flow in an open channel having a trapezoidal cross section (as shown in Figure 1 in Table 1). From Eq. (3), the critical depth $h_{K}$ can be derived. Afterward, the least specific energy of flow in channel $\left(E_{\text {smin }}\right)$ can be calculated using Eq. (1).

However, the conventional method for calculating the specific energy and critical depth of flow is not perfect. From the first derivative method, the extremum cannot be determined directly. Mathematically, by determining the zero value of the first derivative of a function, one can only get the stationary point of a function. However, the stationary point of a function does not mean that the extremum value exists at this point. To determine the point with extreme value, one has to find the point with zero value of the first derivative of a function in addition to finding the points where the first derivative is absent. In the mean time, the changes of the first derivative besides the stationary points of a function should be assessed, or to determine the extremum by means of the second derivative of a function. The calculation of extreme value of a function needs the assessment of point, which is extremum of each stationary maximum. It is a cumbersome process.

In addition, the conventional methods for calculating the specific energy and critical depth of flow do not consider all factors that affect the specific energy and flow depth. For cross sections having complex shapes, it is really difficult to determine the specific energy and critical depth accurately using the conventional method.

\section{CALCULATION OF THE CRITICAL DEPTH AND THE LEAST SPECIFIC ENERGY BY MEANS OF THE ALGEBRAIC INEQUALITY THEORY} Theory of arithmetic-geometric average inequality

Arithmetic-geometric average inequality (AM-GM inequality) is defined as following, for a list of $n$ non-negative real numbers $x_{1}, x_{2}, \ldots, x_{n}$, the arithmetic mean $\left(A_{n}\right)$ of these nonnegative real numbers is greater or equal to the geometric mean $\left(G_{n}\right)$ of the same list, namely, $A_{n} \geq G_{n}$ (Garling, 2012).

The arithmetic mean $\left(A_{n}\right)$ can be determined as following

$A_{n}=\frac{1}{n} \sum_{i=1}^{n} x_{i}=\frac{x_{1}+x_{2}+\ldots \ldots+x_{n}}{n}$

and the geometric mean $\left(G_{n}\right)$ can be determined as following

$G_{n}=\sqrt[n]{\prod_{i=1}^{n} x_{i}}=\sqrt[n]{x_{1} x_{2} \ldots \ldots x_{n}}$

The equal sign in the AM-GM inequality $A_{n} \geq G_{n}$ is valid if and only if $x_{1}=x_{2}=, \ldots,=x_{n}$.

There are many methodologies for proving the AM-GM inequality (Garling, 2012). The AM-GM inequality is a classical inequality and has been widely used in mathematics. Up to date, much research has been conducted to apply the AM-GM inequality in engineering designs (Sabnis and Agnihothram, 2006).

There is a similar inequality for the weighted arithmetic average mean and weighted geometric average mean (Furuichi, 2011). Specifically, let the non-negative numbers $x_{1}, x_{2}, \ldots, x_{n}$ and the non-negative weights $p_{\mathrm{i}}>1(\mathrm{i}=, 1,2,3, \ldots, \mathrm{n})$ be given. Set $\sum_{i=1}^{n} 1 / p_{i}=1$. Then, the inequality

$$
\sum_{i=1}^{n} \frac{1}{p_{i}} x_{i} \geq \prod_{i=1}^{n}\left(x_{i}\right)^{1 / p_{i}}
$$

Inequality (6) holds with equality if and only if $x_{1}=x_{2}=\ldots$, $=x_{n}$. In the next sections, Inequality (6) will be used to derive formulas for calculating the critical depth and specific energy of flow in channels with some specific cross-sectional shapes.

\section{Equations for critical depth and specific energy derived based on AM-GM inequality}

Mathematically, if one equation can be solved through the general arithmetic rules and has at least one solution, this equation can be defined as an equation having an analytical solution. In open channel hydraulics, equations for calculating critical depth have analytical solutions. The relationship between flow cross-sectional area $(A)$ and flow depth $(h)$ can be described as following,

$$
A=p(h+q)^{r}
$$

where, $p, q$ and $r$ are constants and have nothing to do with flow depth $(h)$; both $q$ and $h$ have the same dimension of length (m); $r$ is a dimensionless constant; the dimension of $p$ depends on the value of " $r$ " $\left(\mathrm{m}^{2-r}\right)$. Then, the analytical solution for the critical depth can be determined by means of the theory of weighted mean inequality.

Combining Eq. (1) with Eq. (7), Eq. (8) can be derived as,

$$
E_{s}=h \cos \theta+\frac{\alpha Q^{2}}{2 g p^{2}(h+q)^{2 r}}
$$

As shown in Eq. (8), to obtain the least specific energy, the right hand side of Eq. (8) should be transformed. Applying the method of the weighted mean inequality, variable $h$ can be eliminated. Then, under condition of equality holding, the right hand side of Eq. (8) can be split into several parts. After identical transformation, Eq. (8) can be changed as follows,

$$
E_{S}=2 r\left[\frac{(h+q)}{2 r} \cos \theta\right]+\frac{\alpha Q^{2}}{2 g p^{2}(h+q)^{2 r}}-q \cos \theta
$$

From the weighted mean inequality (6), we obtain the following,

$$
\begin{aligned}
& \frac{2 r}{2 r+1}\left[\frac{(h+q)}{2 r} \cos \theta\right]+\frac{1}{2 r+1}\left[\frac{\alpha Q^{2}}{2 g p^{2}(h+q)^{2 r}}\right] \\
& \geq\left[\frac{(h+q)}{2 r} \cos \theta\right]^{\frac{2 r}{2 r+1}}\left[\frac{\alpha Q^{2}}{2 g p^{2}(h+q)^{2 r}}\right]^{\frac{1}{2 r+1}} \\
& =2 r+1 \sqrt{\left[\frac{\cos \theta}{r}\right]^{2 r} \frac{\alpha Q^{2}}{p^{2} g}} / 2
\end{aligned}
$$

If both sides are multiplied by $(2 r+1)$, Eq. (9) can be expressed as follows, 


$$
\begin{aligned}
& 2 r\left[\frac{(h+q)}{2 r} \cos \theta\right]+\frac{\alpha Q^{2}}{2 g p^{2}(h+q)^{2 r}}-q \cos \theta \\
& \geq \frac{(2 r+1)}{2} \sqrt[r+1]{\left[\frac{\cos \theta}{r}\right]^{2 r} \frac{\alpha Q^{2}}{p^{2} g}-q \cos \theta}
\end{aligned}
$$

Thus, the specific energy of flow at the designated cross section can be calculated from the following equation,

$$
E_{s \min }=\frac{(2 r+1)}{2} \sqrt[r+1]{\left[\frac{\cos \theta}{r}\right]^{2 r} \frac{\alpha Q^{2}}{p^{2} g}}-q \cos \theta
$$

Inequality (11) holds with equality if the flow has the least specific energy. Thus, considering the case when weighted mean inequality holds with equality, we obtain the following,

$$
\frac{(h+q)}{2 r} \cos \theta=\frac{\alpha Q^{2}}{2 g p^{2}(h+q)^{2 r}}
$$

Then the equation for the critical depth of flow can be determined as follows,

$$
\frac{\left(h_{K}+q\right)}{2 r} \cos \theta=\frac{\alpha Q^{2}}{2 g p^{2}\left(h_{K}+q\right)^{2 r}}
$$

From Eq. (14), the calculation of the critical depth of flow at a designated cross section $\left(h_{K}\right)$ can be expressed as follows,

$$
h_{K}=\sqrt[2 r+1]{\frac{r \alpha Q^{2}}{g p^{2} \cos \theta}}-q
$$

Eq. (15) has been derived provided that the relationship between flow cross-sectional area and flow depth can be described by Eq. (7). The calculated critical flow depth at the designated cross section as defined by Eq. (14) is an analytical solution.

Generally, flow cross sections in natural rivers can be approximated by one of the following shapes: rectangular, (quasi-)trapezoidal, (quasi-)U, or a high-order parabolic shape. The relationship between the cross-sectional flow area and flow depth for all above-mentioned cross-sectional shapes can be described as Eq. (7). Namely, there exist analytical solutions for critical flow depth in channels having above-mentioned crosssectional shapes. In the following sections, equations for determining the critical depth and the least specific energy for natural channels having such typical cross-sectional shapes will be derived.

\section{Solving equations for determining critical depth and specific energy for steady non-uniform flow}

Mathematically, the following methodologies can be used to get numerical solutions of equations by means of numerical analysis, iterative method, numerical step-by-step method, and interpolation method. One can calculate the critical depth of flow by means of numerical analysis provided the relationship between flow cross-sectional area and flow depth can be described as follows,

$$
A=p \prod_{i=1}^{n}\left(h+q_{i}\right)^{r_{i}}, \quad(\mathrm{n} \geq 2)
$$

where, $p, q$ and $r$ are constants and have nothing to do with flow depth (h); both $q_{i}$ and $h$ have the same dimension of length $(\mathrm{m}) ; r$ is a dimensionless constant; the dimension of $p$ depends on the value of " $r$ " $\left(\mathrm{m}^{2-\mathrm{r}}\right)$.

Then, combining Eq. (1) with Eq. (16), the following equation has been derived for determining the specific energy,

$$
E_{s}=h \cos \theta+\frac{\alpha Q^{2}}{2 g p^{2} \prod_{i=1}^{n}\left(h+q_{i}\right)^{2 r_{i}}}
$$

To get the least specific energy from Eq. (17), flow depth $(h)$ is eliminated by means of the method of mean inequality. Then, by holding with equality, the critical depth can be determined. By splitting and identical transforming of the right side of Eq. (17), and through such an inequality minification as Inequality (6), we get following,

$$
\begin{aligned}
& \sum_{i=1}^{n} \frac{2 r_{i}}{\left(\sum_{i=1}^{n} 2 r_{i}+1\right)^{2}}\left[k_{i}\left(h+q_{i}\right) \cos \theta\right]+\frac{1}{\left(\sum_{i=1}^{n} 2 r_{i}+1\right)}\left[\frac{\alpha Q^{2}}{2 g p^{2} \prod_{i=1}^{n}\left(h+q_{i}\right)^{2 r_{i}}}\right] \\
& \left.\geq \prod_{i=1}^{n}\left[k_{i}\left(h+q_{i}\right) \cos \theta\right]^{2 r_{i} /\left(\sum_{i=1}^{n} 2 r_{i}+1\right)}\right]\left[\frac{\alpha Q^{2}}{2 g p^{2} \prod_{i=1}^{n}\left(h+q_{i}\right)^{2 r_{i}}}\right]^{1 /\left(\sum_{i=1}^{n} 2 r_{i}+1\right)} \\
& =\sum_{i=1}^{n} 2 r_{i}+1 \sqrt{\prod_{i=1}^{n}\left[k_{i} \cos \theta\right]^{2 r_{i}} \frac{\alpha Q^{2}}{2 p^{2} g}}
\end{aligned}
$$

When both sides are multiplied by $\sum_{i=1}^{n} 2 r_{i}+1$, we get,

$$
\begin{aligned}
& \sum_{i=1}^{n} 2 r_{i}\left[k_{i}\left(h+q_{i}\right) \cos \theta\right]+\frac{\alpha Q^{2}}{2 g p^{2} \prod_{i=1}^{n}\left(h+q_{i}\right)^{2 r_{i}}} \\
& \geq\left(\sum_{i=1}^{n} 2 r_{i}+1\right) \cdot \sum_{i=1}^{n} 2 r_{i}+1 \sqrt{\prod_{i=1}^{n}\left[k_{i} \cos \theta\right]^{2 r_{i}} \frac{\alpha Q^{2}}{2 p^{2} g}}
\end{aligned}
$$

Eq. (17) can be modified as following,

$$
\begin{aligned}
& E_{S}=\sum_{i=1}^{n} 2 r_{i}\left[k_{i}\left(h+q_{i}\right) \cos \theta\right]+\frac{\alpha Q^{2}}{2 g p^{2} \prod_{i=1}^{n}\left(h+q_{i}\right)^{2 r_{i}}} \\
& -2 \sum_{i=1}^{n} q_{i} r_{i} k_{i} \cos \theta \geq\left(\sum_{i=1}^{n} 2 r_{i}+1\right) \sum_{i=1}^{n} 2 r_{i}+1 \sqrt{\prod_{i=1}^{n}\left(k_{i} \cos \theta\right)^{2 r_{i}} \frac{\alpha Q^{2}}{2 p^{2} g}} \\
& -2 \sum_{i=1}^{n} q_{i} r_{i} k_{i} \cos \theta=E_{\text {s min }}
\end{aligned}
$$

where, $k_{i}(i=1,2, \ldots, n)$ in inequality (20) are un-determined dimensionless coefficients. Since inequality (20) is the identical transformation of the right hand side of Eq. (17), therefore,

$2 \sum_{i=1}^{n} r_{i} k_{i}=1$ 
According to conditions for holding with equality of the weighted mean inequality, the following equation was obtained,

$$
k_{i}\left(h+q_{i}\right) \cos \theta=k_{j}\left(h+q_{j}\right) \cos \theta=\frac{\alpha Q^{2}}{2 g p^{2} \prod_{i=1}^{n}\left(h+q_{i}\right)^{2 r_{i}}}
$$

In Eq. (22), $i \neq j$. Combining Eq. (21) with Eq. (22), coefficients $k_{i}(i=1,2, \ldots, n)$ can be determined. By holding with equality, $E_{s}=E_{\min }$, and thus, $h=h_{k}$. Letting the un-determined coefficients $k_{i}(i=1,2, \ldots, n)$ to be following,

$k_{i}=\psi_{i}\left(h_{K}\right)$

Considering convergence speed of iterative solution, with $\max _{1 \leq i \leq n}\left\{r_{i}\right\}=r_{M}$, Eq. (24) was derived,

$k_{M}\left(h_{K}+q_{M}\right) \cos \theta=\frac{\alpha Q^{2}}{2 g p^{2} \prod_{i=1}^{n}\left(h_{K}+q_{i}\right)^{2 r_{i}}}$

Combining Eq. (23) with Eq. (24), resulted in Eq. (25),

$$
\begin{aligned}
& h_{K}=\sqrt[2 r_{M}+1]{\frac{\alpha Q^{2}}{2 g p^{2} \cos \theta \prod_{i \neq M}^{n}\left(h_{K}+q_{i}\right)^{2 r_{i}} \psi_{M}\left(h_{K}\right)}}-q_{M} \\
& \text { Let } \varphi(x)=\sqrt[2 r_{M}+1]{\frac{\alpha Q^{2}}{2 g p^{2} \cos \theta \prod_{i \neq M}^{n}\left(x+q_{i}\right)^{2 r_{i}} \psi_{M}(x)}}-q_{M} .
\end{aligned}
$$

From Eq. (25), one can see that $h_{K}$ can be described as $h_{K}=\varphi\left(h_{K}\right)$. Thus, $h_{K}$ is the fixed point of this function equation. The fixed point iterative formula for the critical depth is $h_{K, s+1}=\varphi\left(h_{K, s}\right)$, where $s$ represents the iteration number $(s=$ $1,2, \ldots$, ). Thus,

$$
h_{K, s+1}=\sqrt[2 r_{M}+1]{\frac{\alpha Q^{2}}{2 g p^{2} \cos \theta \prod_{i \neq M}^{n}\left(h_{K, s}+q_{i}\right)^{2 r_{i}} \psi_{M}\left(h_{K, s}\right)}}-q_{M}
$$

After successive iteration of Eq. (26), the $\left\{h_{K, s}\right\}$ series will be determined. Then, $\lim _{s \rightarrow \infty} h_{K, s}=h_{K}$. Thus, the critical depth can be determined using the fixed-point iteration. Afterward, the least specific energy can be calculated using Eq. (1). To speed up the iterative convergence, the iterative method proposed by Kincaid and Cheney (2003) can be used. Critical depth calculated using Eq. (26) is the numerical solution for open channel flows (in these open channel flows, the relationship between cross-sectional area and flow depth can be described by Eq. (16)).

In practice, the cross sections of natural rivers have either trapezoidal or $U$ shapes. The relationship between crosssectional area and flow depth for channels having both trapezoidal and U-shapes can be described by Eq. (16). For other complicated cross-sectional shapes, by means of some approximation methods such as interpolation, the relationships between cross-sectional area and flow depth can be also described by Eq. (16). The theory of algebraic inequality can also be used to solve the critical flow depth.

In the following section, we take the trapezoidal cross section shape and derive the hydraulic calculation of the critical depth and the least specific energy of flows by means of algebraic inequality. For flow in channels having other common cross- sectional shapes, a similar calculation method will be used to determine the critical depth and the least specific energy, as shown in Table 1.

\section{Formulas for hydraulic calculation of flow in channel having a trapezoidal cross section}

In river engineering, the cross section of natural channels has been often assumed to have a trapezoidal shape. As shown in Figure 1, the width of channel bottom is $b$; the width of water surface is $B ; m_{1}$ and $m_{2}$ represents the left side slope of and right side slope of channel banks, respectively.

The relationship between cross-sectional area and flow depth for the channel having a trapezoidal cross-sectional shape can be written as follows,

$$
A=\frac{1}{2} h(B+b)=\frac{\left(m_{1}+m_{2}\right)}{2} h\left[h+\frac{2 b}{\left(m_{1}+m_{2}\right)}\right]
$$

Comparing Eq. (27) to Eq. (16), $p=\left(m_{1}+m_{2}\right) / 2, q_{1}=0 ; r_{l}=$ $1, q_{2}=2 b /\left(m_{1}+m_{2}\right)$, and $r_{2}=1$. Combining Eq. (21) and Eq. (22) with Eq. (27), then,

$$
\begin{aligned}
& k_{1}=\psi_{1}\left(h_{K}\right)=\frac{h_{K}+2 b /\left(m_{1}+m_{2}\right)}{4\left\{h_{K}+b /\left(m_{1}+m_{2}\right)\right\}} \\
& k_{2}=\psi_{2}\left(h_{K}\right)=\frac{h_{K}}{4\left\{h_{K}+b /\left(m_{1}+m_{2}\right)\right\}}
\end{aligned}
$$

From $\max _{1 \leq i \leq n}\left\{r_{i}\right\}=r_{M}=r_{1}=1, q_{M}=q_{1}=0$ and Eq. (26), the formula for determining the critical flow depth is derived as following,

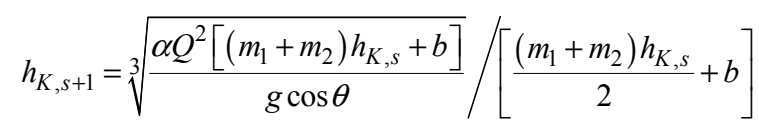

When $m_{1}=m_{2}=0$, the shape of trapezoidal cross section becomes a rectangular cross-sectional shape. When $b=0$, the trapezoidal cross-sectional shape becomes to a triangular crosssectional shape. This means, critical flow depths for flows in channels having cross sections of both rectangular and triangular shapes can be calculated using Eq. (29). The derived formulas for specific conditions (such as either " $m_{1}=m_{2}=0$ " or " $b=$ 0 ") for determining critical flow depths from Eq. (29) are identical to those for flows in channels having cross sections of both rectangular and triangular shapes, respectively ( $\mathrm{Wu}, 2008)$. This proves the accuracy of Eq. (29).

As summarized in Table 1, formulas for determining the critical depth and the least specific energy of flow in channels having other typical cross-sectional shapes have been also derived. 
Table 1. Formulas for calculating critical depth of steady non-uniform flow $\left(h_{K}\right)$ in open channel developed by the method of algebraic inequality.

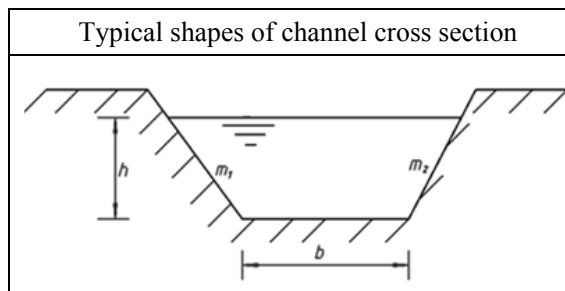

Fig. 1. Diagram of channel having a trapezoidal cross-sectional shape.

\section{\begin{tabular}{|c|c|c}
\hline Cross-sectional area $(A)$ & Values of $p, q, r$ \\
$A=$ & $p=\frac{\left(m_{1}+m_{2}\right)}{2}$ \\
$\frac{\left(m_{1}+m_{2}\right)}{2} h\left[h+\frac{2 b}{\left(m_{1}+m_{2}\right)}\right]$ & $q_{1}=0, r_{1}=1$ \\
$q_{2}=\frac{2 b}{\left(m_{1}+m_{2}\right)}, \quad r_{2}=1$
\end{tabular}}

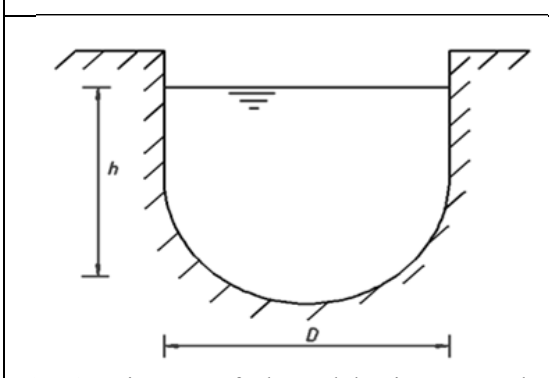

Fig. 2. Diagram of channel having a U- shape cross-sectional shape.

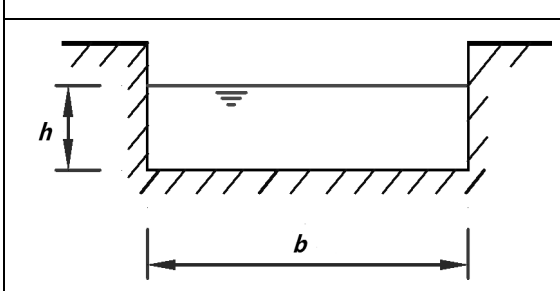

Fig. 3. Diagram of channel having a rectangle cross-sectional shape.

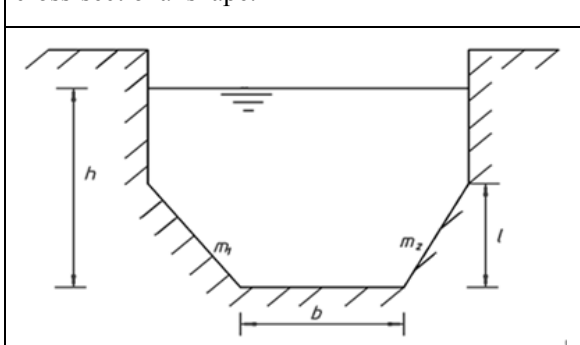

Fig. 4. Diagram of channel having a quasi trapezoid shape cross-sectional shape.

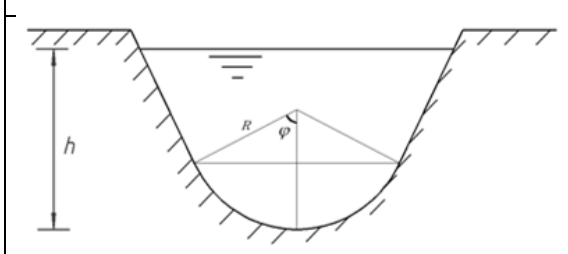

Fig. 5. Diagram of channel having a quasi-Ushape cross-sectional shape.

$$
\begin{aligned}
& A= \\
& {\left[b+\left(m_{1}+m_{2}\right) l\right] *} \\
& \left\{h-\frac{\left(m_{1}+m_{2}\right) l^{2}}{2\left[b+\left(m_{1}+m_{2}\right) l\right]}\right\}
\end{aligned}
$$$$
A=D\left[h-\frac{D}{8}(4-\pi)\right]
$$$$
p=D
$$$$
q=-\frac{D}{8}(4-\pi)
$$$$
r=1
$$

Critical flow depth $\left(h_{K}\right)$

Numerical solution:

$$
h_{K, s+1}=
$$

$$
\frac{\sqrt[3]{\frac{\alpha Q^{2}\left[\left(m_{1}+m_{2}\right) h_{K, s}+b\right]}{g \cos \theta}}}{\left[\frac{\left(m_{1}+m_{2}\right) h_{K, s}}{2}+b\right]}
$$

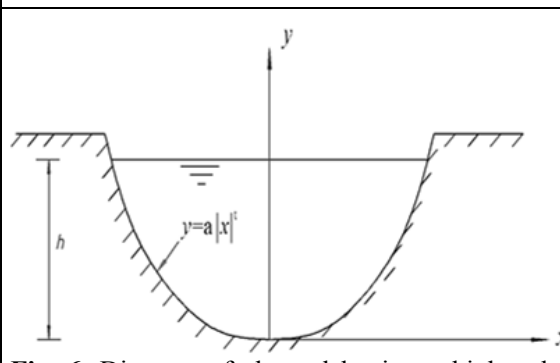

Fig. 6. Diagram of channel having a high-order parabolic cross-sectional shape. 


\section{Calculation examples}

Example 1: In an open channel having a trapezoidal cross section as shown in Figure 1, since the soil in the left bank of the channel is different from that in right bank of the channel, the side slope of the left bank is $m_{1}=1.5$, and the side slope of the right bank is $m_{2}=1.0$, respectively. The width of channel bottom is $b=6 \mathrm{~m}$. The designed flow discharge is $Q=54$ $\mathrm{m}^{3} / \mathrm{s}$. The slope of channel bed is $i=0.008$. The kinematic energy coefficient $\alpha=1.0$. Determine the critical flow depth $\left(h_{K}\right)$ and the least specific energy $\left(E_{\text {smin }}\right)$.

Solution: the channel slope is 0.008 , thus, $\cos \theta=$ $1 /(1+i)^{0.5}$. For flow in an open channel having a trapezoidal cross section, the critical flow depth can be calculated using Eq. (29),

$h_{K, s+1}=\sqrt[3]{\frac{1.0 \times 54^{2}\left[(1.5+1.0) h_{K, s}+6\right]}{9.8 \times 1 / \sqrt{1+0.008^{2}}}} /\left[\frac{(1.5+1.0) h_{K, s}}{2}+6\right]$

Letting $h_{K, 0}=0$, from Table $1, h_{K, 1}=2.022 \mathrm{~m}, h_{K, 2}=$ $1.744 \mathrm{~m}, h_{K, 3}=1.779 \mathrm{~m}, h_{K, 4}=1.775 \mathrm{~m}$, and $h_{K, 5}=1.775 \mathrm{~m}$.

Thus, the critical flow depth $h_{K}=1.775 \mathrm{~m}$.

Using Eq. (3), the hydraulic calculation can be checked:

$\frac{A_{K}^{3} \cos \theta}{B_{K}}=297.44$

$\frac{\alpha Q^{2}}{g}=297.55$

One can see that the relative error using our proposed method is just: $\frac{297.55-297.44}{297.55} \times 100 \%=0.037 \%$

From the given data, the discharge per unit width is $q=Q / b$ $=9 \mathrm{~m}^{3} / \mathrm{s} / \mathrm{m}$, the average side slope of channel is $m=\left(m_{1}+m_{2}\right) / 2$ $=1.25$, the dimensionless factor is $k=\frac{4 m}{b} \sqrt[3]{\frac{\alpha q^{2}}{g}}=1.685 ;$ letting $x=\frac{m h_{K}}{b}$.

In Table 2, the calculated result using our proposed method is compared to five other methods. One can see from Table 2 that the calculation accuracy using our proposed method $(0.037 \%)$ is much higher than those of others.

After determining the critical flow depth $\left(h_{K}\right)$, the least specific energy $\left(E_{\text {smin }}\right)$ can be calculated using either Eq. (1) or Eq. (20),

$$
E_{s \min }=h_{K} \cos \theta+\frac{\alpha Q^{2}}{2 g\left\{\frac{\left(m_{1}+m_{2}\right)}{2}\left[h_{K}+\frac{2 b}{\left(m_{1}+m_{2}\right)}\right] h_{K}\right\}^{2}}
$$

$=2.474 \mathrm{~m}$

Thus, using our formulas, the critical flow depth is $h_{K}=1.775 \mathrm{~m}$, and the least specific energy $E_{\text {smin }}=2.474 \mathrm{~m}$, respectively.

Example 2: In an open channel having U-shape cross section as shown in Figure 2, the flow has a designed discharge of $Q=45 \mathrm{~m}^{3} / \mathrm{s}, D=5.0 \mathrm{~m}$. The channel bed is horizontal. The kinematic energy coefficient $\alpha=1.1$. Determine the critical flow depth $\left(h_{K}\right)$ and the least specific energy $\left(E_{\text {smin }}\right)$.

Solution: the channel slope is 0 , thus, $\cos \theta=1$. For the flow in an open channel having U-shape cross section, as shown in Table 1 , the critical flow depth $\left(h_{K}\right)$ and the least specific energy $\left(E_{\text {smin }}\right)$ can be calculated as follows,

$$
\left\{\begin{array}{l}
E_{s \text { min }}=\frac{3}{2} \sqrt[3]{\frac{1.1 \times 45^{2}}{5^{2} \times 9.81}}+\frac{5 \times(4-\pi)}{8}=3.666 \mathrm{~m} \\
h_{K}=\sqrt[3]{\frac{1.1 \times 45^{2}}{5^{2} \times 9.81}}+\frac{5 \times(4-\pi)}{8}=2.623 \mathrm{~m}
\end{array}\right.
$$

Table 2. Comparison of calculated critical depth of steady non-uniform flow $\left(h_{K}\right)$ in open channel using different formulas proposed by

\begin{tabular}{|c|c|c|c|c|}
\hline Formulas proposed by & $x$ value & & $h_{K}(\mathrm{~m})$ & Relative error $(\%)$ \\
\hline Prabhata & $x=\left[\left(\frac{k}{4}\right)^{-2.1}+\left(\frac{k^{3}}{32}\right)^{-0.42}\right]^{0.476}=0.364$ & & 1.746 & 5.39 \\
\hline Prabhata and Pushpa & $x=\left[\begin{array}{l}\left(\frac{3.1748}{k}\right)^{0.6}+\left(\frac{1.78179}{k}\right)^{1.2}+\left(\frac{0.88341}{k}\right)^{1.8} \\
-\left(\frac{0.68263}{k}\right)^{2.4}-\left(\frac{0.73686}{k}\right)^{3}+\left(\frac{0.72866}{k}\right)^{3.6} \\
+\left(\frac{0.77988}{k}\right)^{4.2}-\left(\frac{0.79676}{k}\right)^{4.8}-\left(\frac{0.83631}{k}\right)^{5.4}\end{array}\right.$ & $=0.373$ & 1.788 & 2.47 \\
\hline Wang & $x=\frac{\sqrt{1+k(k+1)^{0.2}}-1}{2}=0.374$ & & 1.793 & 3.53 \\
\hline $\mathrm{Wu}$ and Patopodis & $x=0.55 k^{0.59}-0.11 \operatorname{arsh}(9.75 k)=0.364$ & & 1.747 & 5.21 \\
\hline Zhao & $x=\frac{\sqrt{1+k\left(k^{1.2}+1\right)^{1 / 6}}-1}{2}=0.367$ & & 1.763 & 2.31 \\
\hline
\end{tabular}
other researchers. 
The calculated critical flow depth of $h_{K}=2.623 \mathrm{~m}$, and the least specific energy of $E_{\text {smin }}=3.666 \mathrm{~m}$ are the exact solutions.

One can see that, it is a practical method to apply the theory of algebraic inequality for determining the critical depth and the least specific energy of flow. The calculation process is straightforward and convenient. The calculation results using our proposed formula are more accurate than those using other methods.

\section{CONCLUSIONS}

In this paper, a new technique based on the theory algebraic inequality has been developed to determine the critical depth and the least specific energy of the steady non-uniform flow in open channel. By means of the theory of weighted arithmetic average mean and weighted geometric average mean, the iterative formulas for calculating the critical flow depth and the least specific energy have been improved. Assessment of the relationships between flow cross-sectional area and flow depth for the typical cross-sectional shapes indicate that there exists analytical solution and numerical solution of equation for determining critical depth. In our proposed new technique for determining the critical depth and the least specific energy of energy, the complicated and cumbersome derivative calculation process of the conventional method has been avoided. Instead of using the time-consuming trail-and-error method, one can calculate the critical flow depth and the least specific energy directly. The calculation process of this new technique is conscious and convenient. Two examples are given to test the calculation results of the critical depth and the least specific energy of flows in channels having the most common crosssectional shapes, namely, trapezoidal and U-shapes. The comparison of calculation results using this new technique to those using other conventional methods has been conducted. Results show that the proposed new method for determining the critical depth and the least specific energy of a steady non-uniform flow is a straightforward and easy calculation process, giving the best results. The method of algebraic inequality can be used in the hydraulic analysis and calculation, and provide better results in engineering practice.

Acknowledgement. This study is supported by the National Natural Science Foundation of China (Funding Number: 51379054). The authors are grateful for the financial support.

\section{REFERENCES}

Furuichi, S., 2011. On refined Young inequalities and reverse inequalities. Journal of Mathematical Inequalities, 5, 21-31.

Garling, D.J.H., 2012. Inequalities: A Journey into Linear Analysis. Beijing World Book Press, Beijing.

Kincaid D., Cheney W., 2003. Numerical Analysis. Beijing Machinery Industry Press, Beijing.

Li, F., Wen, H., Chen, X., 2010. Explicit formula of hydraulic; calculation of U-shaped channel. Advances in Science and Technology of Water Resources, 30, 1, 65-67. (In Chinese.)

Liu, S., Xue, J., 2016. Theoretical analysis and numerical simulation of mechanical energy loss and wall resistance of steady open channel flow. Journal of Hydrodynamics, 28, 3, 489-496.

Sabnis, S.V., Agnihothram, G., 2006. Application of arithmetic -geometric mean inequality for construction of reliability test plan for parallel systems in the presence of covariates. Economic Quality Control, 21, 2, 219-230.

Swamee, P.K., 1993. Critical depth equations for irrigation canals. Journal of Irrigation and Drainage Engineering, ASCE, 119, 2, 400-409.

Swamee, P.K., Rathie, P.N., 2005. Exact equations for critical depth in a trapewidal canal. Journal of Irrigation and Drainage Engineering, ASCE, 131, 5, 474-476.

Swamee, P. K., Wu, S., Katopodis, C., 1999. Formula for calculating critical depth of trapezoidal open channel. Journal of Hydraulic Engineering, ASCE, 125, 7, 785-786.

Vatankhah, A.R., 2013. Multiple critical depth occurrence in two-stage cross sections: effect of side slope change. ASCE Journal of Hydrologic Engineering, 18, 6, 722-728.

Wang, Z., 1998. Formula for calculating critical depth of trapezoidal open channel. Journal of Hydraulic Engineering, ASCE, 124, 1, 90-92.

Wu, C., 2008. Hydraulics (Vol. 1). Beijing Higher Education Press, Beijing. (In Chinese.)

Zhang, Z., Li, R., 2012. Research on critical water depth, Froude Number and hydraulic jump of U-shaped channel. Journal of Xi'an University of Technology, 28, 2, 198-202. (In Chinese.)

Zhao, Y., Zhu, H., Song, S., 2009. Discuss on accurate calculation formula of critical depth of open trapezoidal channel. Journal of Yangtze River Scientific Research Institute, 2009, 04, 18-21+47. (In Chinese.) 